\title{
Le Rapport Taylor de 2017
}

Jo Carby-Hall

\section{(2) OpenEdition}

\section{Journals}

Édition électronique

URL : https://journals.openedition.org/rdctss/2061

DOI : 10.4000/rdctss.2061

ISSN : 2262-9815

\section{Éditeur}

Centre de droit comparé du travail et de la sécurité sociale

\section{Édition imprimée}

Date de publication : 1 avril 2018

Pagination : 182-185

ISSN : 2117-4350

\section{Référence électronique}

Jo Carby-Hall, « Le Rapport Taylor de 2017 », Revue de droit comparé du travail et de la sécurité sociale [En ligne], 1 | 2018, mis en ligne le 01 novembre 2021, consulté le 13 novembre 2021. URL : http:// journals.openedition.org/rdctss/2061 ; DOI : https://doi.org/10.4000/rdctss.2061

\section{(c) (i) (9)}

Revue de droit comparé du travail et de la sécurité sociale est mise à disposition selon les termes de la Licence Creative Commons Attribution - Pas d'Utilisation Commerciale - Pas de Modification 4.0 International. 


\section{JO CARBY-HALL}

UNIVERSITÉ DE HULL

\section{LE RAPPORT TAYLOR DE 2017}

La démarche du Rapport Taylor se base sur une seule ambition: «Tout travail réalisé dans l'économie britannique doit être juste et honnête, donner des perspectives réalistes de développement et d'épanouissement personnels ». Un bon emploi est important car (a) "l'équité requiert que toute personne, celles qui ont de bas salaires en particulier, puisse progresser dans son travail, ait l'occasion d'améliorer son pouvoir d'achat et soit traitée avec respect et décence au travail »; (b) « la qualité de l'emploi est un facteur primordial de santé et de contentement personnels qui profite au travailleur et qui sert l'intérêt public en général »; (c) un travail mieux pensé qui améliore le rendement du travailleur peut apporter une contribution significative au défi complexe posé par notre bas taux de productivité »; (d) « un principe fondamental devrait être que l'expérience professionnelle du travailleur soit à la hauteur des aspirations de chaque citoyen moderne, que les personnes éprouvent le respect, la confiance et la possibilité d'assumer des responsabilités » et (e) que «les changements rapides dans l'économie moderne, en particulier dans le domaine des technologies et le développement de nouveaux modèles d'affaires nécessitent une approche concertée du travail ${ }^{1}$. Malheureusement, nombreux sont les exemples d'exploitation des travailleurs vulnérables par le patronat. Voilà donc de beaux principes auxquels les relations industrielles britanniques devraient aspirer. Nous n'aborderons dans ce commentaire que certains aspects du rapport Taylor: le statut de l'emploi, la notion de travailleur dépendant, les conditions d'emploi, la flexibilité sur le marché du travail britannique, la continuité de l'emploi, la bonne gestion des entreprises et le dialogue social et la transparence dans les entreprises.

\section{I - LE STATUT DE L'EMPLOI}

Le statut de l'emploi est «le portail par lequel l'individu doit passer pour accéder aux droits légaux». Un des problèmes posés par le statut de l'emploi c'est lorsqu'une personne n'est «ni salarié ni travailleur indépendant» mais jouit du statut de «travailleur ». C'est une des conséquences des innovations technologiques et numériques. Bien que les auteurs du Rapport estiment que le cadre législatif actuel fonctionne correctement et qu'il est suffisamment flexible, ils ont néanmoins affirmé qu'il était nécessaire de clarifier la notion de statut de l'emploi. La démarche actuelle adoptée par les tribunaux et les cours de justice est de déterminer la catégorie dans laquelle se trouve un individu, à savoir salarié, travailleur ou travailleur indépendant. Comme les éléments clé de la relation entre les parties ne sont pas définis par la législation, ils le font en appliquant les principes généraux du contrat en Grande-Bretagne, les Directives de l'UE et une pléthore d'affaires en Common law résolues

1 Source:"Good Work. The Taylor Review of Good Working Practices" July 2017. 
par les tribunaux depuis plus de cent ans ${ }^{2}$. II ressort du Rapport qu'il faudrait modifier la législation pour qu'elle reflète les caractéristiques du statut de l'emploi à travers une législation de base qui « donnerait une réponse dynamique à l'évolution des conditions et au droit jurisprudentiel ; les détails qui étayent ces critères doivent être spécifiés de manière à pouvoir être mis en lumière rapidement par l'utilisation accrue de la législation secondaire et des conseils $»^{3}$. Selon le Rapport Taylor, le statut de l'emploi (employment status) doit être bien distinct et ne pas être si complexe que seul un tribunal puisse en comprendre les principes de base. Si les personnes qui travaillent sont soumises à un contrôle significatif (a significant control) de l'employeur, il ne faut pas qu'elles manquent de protection en raison du libellé attribuée au contrat. II ne faut pas non plus que le statut de l'emploi soit difficile à interpréter par tout un chacun et par les employeurs responsables.

\section{II - RECOMMANDATION SUR LA NOTION DE «TRAVAILLEUR DÉPENDANT"}

Selon le Rapport Taylor, l'actuel système à trois niveaux - travailleur indépendant, salarié et travailleur - est déroutant et les deux catégories «d'ayants-droit» devraient être plus faciles à distinguer l'une de l'autre. Le Rapport recommande donc que le gouvernement introduise une nouvelle appellation qui désigne la catégorie de personnes ayant droit à la protection au titre de travailleur - «worker» - mais qui ne sont pas des salariés - « employees »-. Il suggère que cette catégorie soit appelée: "travailleurs dépendants » (dependent contractors). Il faudrait également que le gouvernement préserve le système des trois niveaux applicable au marché du travail moderne. De plus, grâce au développement du test pour ce statut, le contrôle par l'employeur aura une importance accrue; l'accent sera moins mis sur la nécessité d'accomplir la tâche par la personne elle-même comme c'est le cas en ce moment pour définir le statut de travailleur (worker).

\section{III - DESCRIPTION DE L'EMPLOI}

Au titre de la législation britannique l'employeur n'a pas l'obligation de fournir un contrat au salarié. II doit cependant lui fournir par écrit, dans un délai de deux mois après l'embauche une description détaillée du travail. Le Rapport suggère que le gouvernement introduise une réglementation pour que le salarié et le travailleur dépendant reçoivent les informations sur l'emploi dès le premier jour de travail. Actuellement, les informations qui doivent figurer sur la déclaration écrite sont les suivantes: le nom de l'employeur, le lieu de travail, le salaire, les congés payés, le droit aux allocations maladie et le droit à la retraite. Le Rapport préconise une extension du champ d'application de ces informations

2 Pour une analyse détaillée voir J. Carby-Hall «New Frontiers of Labour Law - Dependent and Autonomous Workers » in B. Veneziani et U. Carabelli (dir.) « Du travail salarié au travail indépendantPermanences et Mutations », European SOCRATES, Programme, Cacucci Editore, 2003, p. 63-287. Du même auteur «Innovative forms pf employment in the 21st Century Versus Employment Status » dans M. Redinha (Rédactrice) «Sociedade de Permitas e Combinaęôes: Problemas Juridicos da Economia Colaborativa ", Cambridge Scholars Publishing à paraître mi-2018 et J. Carby-Hall "The Taylor Review 2017- A Critical Appreciationon on a Selection of its Legal Content» dans Lourdes Mella (Redactrice) Technological Innovations and the Future of Work: Emerging Aspects Worldwide » à paraître fin 2018.

3 "Taylor Review" op. cit., p. 34 - 35. 
aux travailleurs dépendants et comprenant les droits légaux auxquels peuvent aspirer les travailleurs dès le premier jour de travail, le calcul du salaire et les modalités de paiement. De plus, le Rapport suggère que la législation spécifie que ces informations soient données en langue anglaise simple, courante, et accessible. Le Rapport propose que l'employeur qui ne respecte pas ces préceptes soit condamné à payer une somme compensatoire au salarié ou au travailleur dépendant. Une autre suggestion est que le gouvernement crée et développe un outil en ligne d'accès gratuit offrant aux travailleurs les informations sur le statut de l'emploi et sur d'autres questions relatives à l'emploi, mettant ainsi à profit la numérisation pour faciliter l'accès à l'information.

\section{IV - FLEXIBILITÉ DU MARCHÉ DU TRAVAIL AU ROYAUME-UNI}

La nature flexible du marché du travail au Royaume-Uni est bien connue. La flexibilité joue un rôle important et permet aux employeurs de s'adapter aux changements rapides des conditions du marché, tout en favorisant un taux d'emploi élevé. Le Rapport s'inquiète de la manière dont les employeurs utilisent ce concept dans leur seul avantage en exploitant et en contrôlant les travailleurs. C'est dans les affaires Uber, City Sprint et Pimlico Plumbers que ceci est apparu de manière notoire. Ces employeurs ont fait usage de la flexibilité pour imposer à leurs travailleurs le statut de «travailleur indépendant ». Ils contrôlaient la situation en leur imposant un choix: acceptez ou quittez l'emploi. II n'y avait pas la moindre suggestion d'une possibilité de négociation, ce qui équivalait à une flexibilité unilatérale. Une personne vulnérable occupant un emploi atypique, comme par exemple avec les contrats zéro heure et les emplois temporaires (gig-economy) a besoin de protection. La flexibilité du marché du travail est importante comme nous l'avons montré et doit être préservée mais elle ne doit pas être un outil au seul profit d'employeurs peu scrupuleux qui ne se soucient pas de leur main-d'œuvre.

\section{V - LA CONTINUITÉ DE L’EMPLOI}

Le Rapport ne recommande pas l'abolition du concept de continuité d'emploi. Le problème qui se pose pour les personnes qui travaillent avec un contrat zéro heure ou celles qui ont des horaires de travail irréguliers, c'est qu'il peut leur être difficile de faire valoir certains droits en raison de discontinuités dans leur emploi. Pour avoir droit aux indemnités de licenciement, par exemple, ou pour pouvoir intenter une action pour licenciement abusif, un salarié doit avoir eu un emploi continu pendant deux ans. Ceux qui ont des emplois temporaires ou intermittents rencontrent donc des difficultés à prouver la continuité de leur emploi exigée pour bénéficier de droits. Les auteurs du Rapport estiment qu'il y a beaucoup à faire sur ce sujet et proposent d'allonger la période de carence à un mois au lieu d'une semaine. Ainsi, un travailleur occasionnel ayant eu une semaine de travail puis se trouvant sans emploi pour une période maximale d'un mois serait considérée comme ayant eu une continuité d'emploi. 


\section{ROYAUME-UNI}

\section{VI - BONNE GOUVERNANCE DE L'ENTREPRISE ET DIALOGUE SOCIAL}

«Les entreprises bien gérées reconnaissent l'importance de leurs travailleurs et investissent pour établir de bonnes relations entre les individus et au niveau collectif» mentionne le Rapport. Un dialogue social efficace est essentiel pour que le travail soit juste et adéquat; il convient de respecter la dignité des travailleurs, les activités quotidiennes doivent être gérées de manière courtoise et la direction doit considérer comme pertinente et constructive l'opinion des travailleurs sur les méthodes de travail. Les travailleurs, quant à eux, doivent avoir une compréhension générale de la politique de l'entreprise, de ses aspirations et stratégies d'avenir, du rôle des travailleurs et de leur contribution au développement de l'entreprise. Finalement, les travailleurs doivent être informés de décisions majeures de l'entreprise qui les affectent. "La participation des travailleurs et le dialogue social constituent l'essence d'une entreprise bien gérée et de sa réussite ». En outre, la gouvernance de l'entreprise et le dialogue social sont régies par la Common law à laquelle renvoie la législation sur l'emploi. Un grand nombre de ces exigences émanent des Directives de I'Union Européenne transposées dans la législation nationale tandis que d'autres ont leurs sources dans la législation nationale. Citons comme exemple de transposition de la législation européenne dans la législation britannique, la Directive sur les droits acquis (Acquired Rights Directive) ou la Directive sur le transfert d'entreprise (Transfer of Undertakings Directive) qui obligent notamment l'employeur à consulter les représentants des salariés sur divers sujets; il en va de même pour la Directive sur le licenciement collectif pour cause économique (redundancy). Dans la législation nationale, les licenciements individuels sont couverts par l'Employment Rights Act de 1996 qui comprend des dispositions relatives aux négociations d'une convention collective de travail. La Common law britannique contient également des éléments de gouvernance d'entreprise et de dialogue social tels que l'obligation de respect du salarié et l'obligation pour l'employeur de prendre soin des salariés (duty of care). Le Rapport Taylor exprime le désir de voir généraliser à tout le marché du travail, la gouvernance de l'entreprise et le dialogue social. Reste à savoir, si l'on tient compte du Brexit, si la volonté politique du gouvernement et l'agenda parlementaire permettront de légiférer sur toutes ou partie des suggestions du Rapport Taylor. 